\title{
Neuronal Regeneration in C. elegans Requires Subcellular Calcium Release by Ryanodine Receptor Channels and Can Be Enhanced by Optogenetic Stimulation
}

\author{
Lin Sun, ${ }^{1 *} \odot$ James Shay, ${ }^{1 *} \odot$ Melissa McLoed, ${ }^{2}$ Kevin Roodhouse, ${ }^{1}$ Samuel H. Chung, ${ }^{1}$ Christopher M. Clark, ${ }^{3}$ \\ ㄱennifer K. Pirri, ${ }^{3}$ Mark J. Alkema, ${ }^{3}$ and ${ }^{\circledR C}$ Christopher V. Gabel ${ }^{1}$ \\ ${ }^{1}$ Department of Physiology and Biophysics, Photonics Center, Boston University School of Medicine, Boston, Massachusetts 02118, ${ }^{2}$ Department of \\ Pharmacology and Experimental Therapeutics, Boston University School of Medicine, Boston, Massachusetts 02118, and ${ }^{3}$ Department of Neurobiology, \\ University of Massachusetts Medical School, Worchester, Massachusetts 01605
}

\begin{abstract}
Regulated calcium signals play conserved instructive roles in neuronal repair, but how localized calcium stores are differentially mobilized, or might be directly manipulated, to stimulate regeneration within native contexts is poorly understood. We find here that localized calcium release from the endoplasmic reticulum via ryanodine receptor (RyR) channels is critical in stimulating initial regeneration following traumatic cellular damage in vivo. Using laser axotomy of single neurons in Caenorhabditis elegans, we find that mutation of unc-68/RyR greatly impedes both outgrowth and guidance of the regenerating neuron. Performing extended in vivo calcium imaging, we measure subcellular calcium signals within the immediate vicinity of the regenerating axon end that are sustained for hours following axotomy and completely eliminated within $u n c-68 /$ RyR mutants. Finally, using a novel optogenetic approach to periodically photostimulate the axotomized neuron, we can enhance its regeneration. The enhanced outgrowth depends on both amplitude and temporal pattern of excitation and can be blocked by disruption of UNC-68/RyR. This demonstrates the exciting potential of emerging optogenetic technology to beneficially manipulate cell physiology in the context of neuronal regeneration and indicates a link to the underlying cellular calcium signal. Taken as a whole, our findings define a specific localized calcium signal mediated by RyR channel activity that stimulates regenerative outgrowth, which may be dynamically manipulated for beneficial neurotherapeutic effects.
\end{abstract}

Key words: C. elegans; calcium; optogenetics; regeneration; ryanodine receptor

\section{Introduction}

Recent efforts have illustrated the importance of intrinsic cellular and molecular mechanisms in a neuron's ability to regenerate following traumatic damage (Liu et al., 2011). Intracellular calcium signals play a critical role in this process, with excessive calcium overload triggering cell death but more modest signals initiating cellular repair processes and modulating growth cone guidance. Numerous studies in invertebrates (Gitler and Spira, 1998; Ghosh-Roy et al., 2010; Pinan-Lucarre et al., 2012), as well

Received Oct. 1, 2013; revised 0ct. 9, 2014; accepted 0ct. 16, 2014.

Author contributions: L.S., J.S., S.H.C., and C.V.G. designed research; L.S., J.S., M.M., K.R., and C.V.G. performed research; C.M.C., J.K.P., and M.J.A. contributed unpublished reagents/analytic tools; L.S., J.S., M.M., K.R., and C.V.G. analyzed data; C.V.G. wrote the paper.

This work was supported in part by the Massachusetts Life Sciences Center, the National Institute of General Medical Sciences predoctoral training Grant GM008541, National Institute of Neurological Disorders and Stroke Grant R01 NS077929, National Institutes of Health to C.V.G., and Grant GM084491 to M.J.A. Some nematode strains used in this work were provided by the Caenorhabditis Genetics Center, which is funded by the National Institutes of Health National Center for Research Resources. The authors thank C. Reina, M. Driscoll, A. Leifer, and D. Taub for comments and discussions; and D. Austin for advice with MATLAB coding.

The authors declare no competing financial interests.

*L.S. and J.S. contributed equally to this work as co-first authors.

Correspondence should be addressed to Dr. Christopher V. Gabel, Department of Physiology and Biophysics, Photonics Center, Boston University School of Medicine, 72 East Concord Street, Boston, MA 02118. E-mail: cvgabel@bu.edu

DOI:10.1523/JNEUROSCI.4238-13.2014

Copyright $\odot 2014$ the authors $\quad 0270-6474 / 14 / 3415947-10 \$ 15.00 / 0$ as in mammals (Chierzi et al., 2005), demonstrated that neurite transection results in an immediate intracellular calcium response that facilitates cytoskeletal remodeling and growth cone formation. In addition, in vitro studies reveal intricate signaling within an established growth cone whereby asymmetric calcium gradients mediate axon guidance and attractive signals are dependent on amplified calcium release from internal endoplasmic reticulum (ER) stores (Tojima et al., 2011). However, how these internal calcium sources might be involved in the critical transition from immediate damage response to an actively regrowing neurite within its native context remains uncertain.

Using a combination of genetic and in vivo biophotonic techniques, numerous Caenorhabditis elegans studies have identified novel regenerative factors and highlighted the importance of intrinsic cellular calcium signaling. This includes the dual leucine zipper-bearing kinase (DLK-1) that plays a conserved role in regeneration from C. elegans (Hammarlund et al., 2009; Yan et al., 2009) to mammals (Shin et al., 2012). Further work revealed a role of calcium signaling in the activation of DLK-1 (Ghosh-Roy et al., 2010) and a molecular mechanism for this process (Yan and Jin, 2012). C. elegans research has also demonstrated a beneficial role of apoptotic caspases in regeneration, implementing calcium in their activation (Pinan-Lucarre et al., 2012). Furthermore, with its small transparent body, C. elegans enables the application 
of biophotonic techniques that give it a unique advantage for in vivo studies. This includes laser severing of individual axons, subcellular calcium imaging, and, more recently, the potential application of optogenetics to dynamically manipulate single-neuron physiology.

Utilizing the advantages of the C. elegans system, we addressed how cellular calcium signals might stimulate new outgrowth in a damaged neuron. Following earlier findings that release of internal calcium stores is a critical part of the cellular response in a damaged neuron, we focused our efforts on a specific calcium source, the RyR channels, encoded by unc-68 in C. elegans. RyR channels mediate localized calcium release from the ER through a process of calcium-induced calcium release (CICR). Within an established growth cone amplification of calcium gradients via CICR triggers an attractive guidance response and growth cone turning but does not necessarily alter outgrowth rates (Hong et al., 2000). Our results provide a link between this mechanism of axon guidance and a role in initiating regenerative outgrowth in a damaged neuron. By performing in vivo single-neuron laser axotomy, extending subcellular calcium imaging to $5 \mathrm{~h}$ after axotomy and manipulating cell physiology through optogenetic photo-activation, we identify localized RyR channel calcium release as a critical signal in stimulating outgrowth during the initial stages of neuron regeneration.

\section{Materials and Methods}

C. elegans strains, cultivation, and experimental conditions. Strains were cultivated using standard techniques $\left(\right.$ at $20^{\circ} \mathrm{C}$, on NGM agar seeded with Escherichia coli OP50 as a food source). All experiments and laser surgeries were performed with young adult hermaphrodites, with the exception of surgeries on D-type motor neurons that were performed in L4 larval hermaphrodites. Standard genetic techniques were used to generate compound mutant strains (for strain list, see Table 1). Indications in the figures refer to the following strains and conditions. Wild-type:

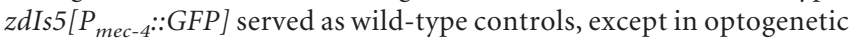
experiments where $p_{m e c-4::} m$ Cherry was used (see Figs. 4 and 5). unc-68: unc-68(e540), channelrhodopsin-2 (ChR2): $P_{\text {mec- } 4:: m \text { Cherry; }}$

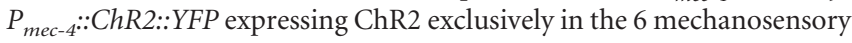
neurons. All-trans retinal (ATR): animals raised overnight in the presence of $10 \mu \mathrm{M}$ ATR. Light: exposure to $470 \mathrm{~nm}$ blue light as indicated. Dant: animals raised overnight in the presence of $10 \mu \mathrm{M}$ dantrolene. NEMA: animals raised on $1 \mu \mathrm{M}$ nemadipine-A. DMSO: animals raised overnight in the presence of DMSO. DMSO serves as the organic solvent for dantrolene and does not affect regeneration at $24 \mathrm{~h}$ (wild-type vs DMSO regeneration was not significantly different by $t$ test). unc-68 rescue lines were generated by injection of the $P_{\text {mec }-3}: R F P$ neuronal marker (and unc-122::RFP as coinjection marker) into unc-68(r1162)V; $z w I s 100[r a b-3 p:: M y c:: R Y R-1+m y o-3 p:: G F P]$; for neuronal expression and unc-68(r1162)V; zwIs108[myo-3p::Myc::RYR-1+myo-3p::GFP]; for muscle expression (Liu et al., 2005). Control experiments with

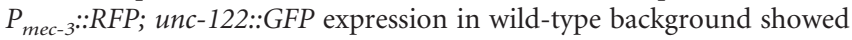
similar $24 \mathrm{~h}$ regeneration to that of $z d I s 5\left[P_{\text {mec }-4:: G F P]}\right.$ (no statistical difference by $t$ test).

Laser surgery and regeneration measurements. Laser surgery was performed as described by Gabel et al. (2008). A Ti:sapphire laser system, Mantis PulseSwitch Laser (Coherent), generated a $1 \mathrm{kHz}$ train of $\sim 100 \mathrm{fs}$ pulses in the near infrared $(\sim 800 \mathrm{~nm})$. The beam, focused to a diffraction limited spot (using a Nikon 60×, 1.4 NA microscope objective) resulted in vaporization and tissue disruption with pulse energies ranging from 5 to $15 \mathrm{~nJ}$. Visual inspection of the targeted neuron immediately following brief laser exposure ( $\sim 100-500 \mathrm{~ms})$ confirmed successful axotomy. In some cases, multiple laser exposures were necessary to generate a visual break in the nerve fiber. For laser surgery and time-lapse microscopy, $C$. elegans were not anesthetized but physically immobilized on a microscope slide using stiff $10 \%$ agarose pads and NGM buffer containing polystyrene beads as described by Kim et al. (2013). For $24 \mathrm{~h}$ measure-
Table 1. List of strains used during this work

\begin{tabular}{|c|c|}
\hline Strain name & Genotype \\
\hline ZB154 & zdls5[Pmec-4::GFP + lin-15(+)]I \\
\hline ZB2691 & zdls5[Pmec-4::GFP]l; unc-68(e540)V \\
\hline CG56 & zdls5[Pmec-4.:GGP] l; unc-68(r1162)V V \\
\hline QW1177 & $\begin{array}{l}\text { unc-68(r1162)V; zwls108[myo-3p:::Myc:::RYR-1 + myo-3p:::GFP]; } \\
\text { zfEx488[Pmec-3::RFP; unc-122::RFP] }\end{array}$ \\
\hline QW1176 & $\begin{array}{l}\text { unc-68(r1162)V;zwls100[rab-3p:::Myc::RYR-1 + myo-3p:::GFP }] \text {; } \\
\text { zfEx487[Pmec-3::RFP; unc-122:::RFP }]\end{array}$ \\
\hline QW1201 & zfEx506[Pmec-3:::RFP; unc-122::GFP] \\
\hline EG1285 & lin-15B(n765); oxls12[Punc-47::GFP + lin-15(+)]X \\
\hline CG57 & $\begin{array}{l}\text { lin-15B(n765); oxls12[Punc-47::GFP + lin-15(+)]X; } \\
\quad \text { unc-68(e540)V }\end{array}$ \\
\hline CG1B & bzls158[Pmec-4::YYC3.60] \\
\hline CG32 & bzls158[Pmec-4::YC3.60]; unc-68(e540)V \\
\hline QW590 & $\begin{array}{l}\text { lin-15(n765ts); zfEx165[Pmec-4::GCaMP3::SL2:::mCherry] (50 } \\
\quad n g / \mu l)\end{array}$ \\
\hline CG45 & $\begin{array}{l}\text { lin-15(n765ts); zfEx165[Pmec-4::GCaMP3::SL2:::mCherry]; } \\
\quad \text { unc-68(e540)V }\end{array}$ \\
\hline KWN177 & myls014[Pmec-4::mCherry + unc-119(+)] \\
\hline CG38 & zfls18[Pmec-4::ChR2::YFP]; myls014[Pmec-4::mCherry $]$ \\
\hline CG43 & $\begin{array}{l}\text { zfls } 18[\text { Pmec-4:::ChR2:::YFP }] ; \text { myls014[Pmec-4::mCherry]; } \\
\text { unc-68(e540)V }\end{array}$ \\
\hline
\end{tabular}

ments, animals were rescued to a standard cultivation agar Petri dish and reimaged using 3 mu sodium azide. The number of neurons axotomized and measured for each condition is indicated in the figures ( $\geq 19$ neurons in each case). Interestingly, $24 \mathrm{~h}$ anterior lateral microtubule (ALM) outgrowth in wild-type was longer than we reported previously using anesthetic (sodium azide) for laser surgery immobilization (PinanLucarre et al., 2012). We targeted ALM and posterior lateral microtubule (PLM) axons $20 \mu \mathrm{m}$ from the cell soma unless otherwise stated. For ALM and PLM $24 \mathrm{~h}$ regeneration assays, neurons were severed in two locations: first at $20 \mu \mathrm{m}$ and then $40 \mu \mathrm{m}$ from the cell soma. The intervening axon segment quickly degenerates, leaving a $20 \mu \mathrm{m}$ gap in the axon that greatly reduced the percentage of regenerating axons that reconnected with the distal disconnected segment ( $<5 \%$ of neurons). Regenerative growth for one neuron was scored as the total length along the contour of all new outgrowth. As previously reported (Pinan-Lucarre et al., 2012), we focused on instances where we could get an accurate measurement of the total length, excluding neurons that appeared to reconnect with the severed distal axon segment and discarding very short branches (those $<5 \mu \mathrm{m}$ for $24 \mathrm{~h}$ measurements and $<1 \mu \mathrm{m}$ for time-lapse measurements). Surgeries on D-type motor neurons were performed in L4 animals by severing the ventral-dorsal commissure $20 \mu \mathrm{m}$ up from the ventral nerve cord.

Statistical analysis. Outgrowth was measured by tracing along all new regenerative branches using ImageJ software (National Institutes of Health). Statistical analysis was performed using Statistical Analysis Systems software version 9.3. Means between different conditions were compared by two-sample $t$ test or one-way ANOVA, with pairwise comparisons tested using a Tukey adjustment. Comparison of percentages for ventral guidance and regeneration initiation point (see Fig. 2) was by Fisher's exact test, and that for behavior (see Fig. 5B) was by logistic regression analysis.

Axon guidance. AVM regeneration assays followed those established by Gabel et al. (2008). The AVM axon was laser severed at a single point halfway down its initial projection to the ventral nerve cord and reimaged at $24 \mathrm{~h}$. The axon guidance angle was measured as the difference in direction between the regenerative outgrowth and the original axon path as shown in Figure $2 A, B$. We also scored the success rate of reaching the ventral nerve cord at $24 \mathrm{~h}$ (see Fig. $2 D$ ). This was higher than reported previously in wild-type possibly because of side effects from sodium azide immobilization in previous experiments. Because the new outgrowth becomes obscured in other labeled neurons once it reaches the ventral nerve cord and the high ventral cord success rate, the total regenera- 
tive outgrowth length could not be accurately determined in AVM neurons.

Calcium imaging. Experiments in Figure $3 A, B$ consisting of short 5 min calcium imaging time-lapse measurements were similar to those by Pinan-Lucarre et al. (2012). Animals expressing cameleon YC3.60 under

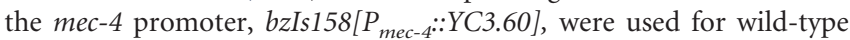
measurements and crossed with unc-68 mutants. Images were taken every $3 \mathrm{~s}$ with a $300 \mathrm{~ms}$ exposure time. The response of an individual neuron was measured as the average fluorescence signal across the cell soma or a region of interest consisting of the axon within $5 \mu \mathrm{m}$ of the damage point. Custom MATLAB software (MathWorks) was used for analysis (for details, see Chung et al., 2013). The software is available upon request and will be made available through sourceforge.net.

Animals expressing $P_{\text {mec- } 4}:: G C a M P 3:: S L 2:: m$ Cherry in the six mechanosensory neurons were used for the extended calcium imaging experiments in Figure $3 C-E$. The coexpression of the two fluorophores allowed us to take advantage of the improved sensitivity of GCaMP3 while using the mCherry as a baseline. This enabled ratiometric measurements that compensate for changes in fluorophore concentration during laser axotomy or over long time periods. Controls that were immobilized but no axotomy performed showed no change in axon fluorescence levels over the $5 \mathrm{~h}$ imaging period (fluorescence at time 0 and time $5 \mathrm{~h}$ were not statistically different by $t$ test). Regenerative outgrowth appeared unaltered in the $5 \mathrm{~h}$ imaging assays for neurons expressing the GCaMP3 construct (45.9 $\pm 8.9 \mu \mathrm{m}$ average outgrowth) and was diminished in unc-68 mutants (19.2 $\pm 4.6 \mu \mathrm{m}$ average outgrowth) consistent with our other results. Single frames with $1 \mathrm{~s}$ exposures were taken at each time point: immediately before laser axotomy, within $20 \mathrm{~s}$ following axotomy and then every hour thereafter. As illustrated in Figure $3 F$, for each frame, fluorescence was measured across the cell soma, in a $20 \mu \mathrm{m}$ section of the axon halfway between the damage point and the cell soma, and within 5 $\mu \mathrm{m}$ of the damage point or end of the regenerating neuron. (Narrow filopodial structures were excluded as they were too small to generate reliable signals.) Fluorescent bleed-through between the two channels was minimal $(<0.4 \%)$ so fluorescent levels were calculated as (GCaMP3background $) /($ mCherry-background) from average measurements within the region of interest and average background measurements (Chung et al., 2013). C. elegans were immobilized using $10 \%$ agarose pads as above with the addition of $0.05 \%$ tetramisole to further immobilize them over the entire $5 \mathrm{~h}$. Single ratiometric images are displayed in Figure $3 F, G$, constructed by aligning the CGaMP3 and mCherry images and measuring the fluorescents level as above on a pixel-by-pixel bases. (Here, calculations were made using $0.75 \times$ background for the mCherry image to avoid division by zero values in the background regions. Images were individually scaled). Although single images are shown as examples, typically multiple images were necessary for different sections of the neuron to ensure accurate focus.

Optogenetic stimulation of neurons. C. elegans with ChR2 expressed in the mechanosensory neurons (zfis $18\left[P_{\text {mec }-4}:\right.$ ChR2::YFP]; myIs014 $\left.\left[P_{\text {mec- } 4:: m C h e r r y}\right]\right)$ were grown overnight on agar Petri dishes containing $10 \mu \mathrm{M}$ ATR, unless otherwise noted. Animals were kept in the dark or under red light illumination for handling and imaging to avoid bleaching of ATR. Following laser surgery, animals were rescued back onto cultivation dishes with $10 \mu \mathrm{M}$ ATR and left in the dark for $90 \mathrm{~min}$ before exposure to the repeating light stimulus. Uniform light stimulus was administered by a collimated LED light source at $470 \mathrm{~nm}$ (Thorlabs), controlled by LabVIEW software (National Instruments) and calibrated to the indicated intensities. Minimal intensities known to elicit optogenetic photo-activation in C. elegans were used (Schultheis et al., 2011) to avoid excessive photo-toxicity from prolonged light exposure. We measured no difference in the average length of individual branches of the regeneration outgrowth across all conditions in Figure $4 C(\sim 20$ $\mu \mathrm{m}$ branches for each, with no statistical difference by one-way ANOVA).

Light exposures consisted of a repeating pattern, $10 \mathrm{~s}$ on $50 \mathrm{~s}$ off, of $470 \mathrm{~nm}$ light at $175 \mu \mathrm{W} / \mathrm{mm}^{2}$ unless otherwise noted. Intensity measurements (see Fig. $4 G$ ) used this temporal pattern of exposure with intensities of $0,125,150,175$, and $200 \mu \mathrm{W} / \mathrm{mm}^{2}$. For frequency measurements (see Fig. $4 H$ ), exposure patterns of $175 \mu \mathrm{W} / \mathrm{mm}^{2}$ light were used that maintained the total light exposure over $24 \mathrm{~h}: 0.5$ flashes $/ \mathrm{min}=20 \mathrm{~s}$ on $100 \mathrm{~s}$ off, 1 flashes $/ \mathrm{min}=10 \mathrm{~s}$ on $50 \mathrm{~s}$ off, 5 flashes $/ \mathrm{min}=2 \mathrm{~s}$ on $10 \mathrm{~s}$ off. Intensity of the constant light was $29 \mu \mathrm{W} / \mathrm{mm}^{2}$ such that total light exposure was the same as the other trials over $24 \mathrm{~h}$. Confirming previous results (Ghosh-Roy et al., 2010), we measured a 32\% reduction in $24 \mathrm{~h}$ ALM regeneration for animals treated with $1 \mu \mathrm{M}$ nemadipine-A (average outgrowths at $24 \mathrm{~h}$ of $123.4 \mu \mathrm{m}$ vs $182.7 \mu \mathrm{m}$ for treated and wild-type animals, respectively, $p<0.05$ by $t$ test). It is important to note that the regeneration defects of controls in Figure 5A (unc-68(e540), dantrolenetreated and nemadipine-A-treated animals) appear to diminish with ChR2 expression and even more so with both ChR2 expression and ATR (compare Fig. $1 C, D$, with Fig. $5 A$ and data above). Similar effects in long-term applications of ChR2 in C. elegans have been noted in previous experiments (Schultheis et al., 2011) and might result from small changes in baseline potential and/or calcium levels due to the presence of ChR2. Although this raises the possibility of confounding consequences from the ChR2 expression, in all cases photo-activation of ChR2 with light nonetheless fails to enhance regeneration beyond the inactivated levels, suggesting that UNC-68/RyR and EGL-19/VGCC play a role in the enhanced regeneration effect.

A behavioral response to ChR2 activation of ALM neurons was scored as an initiation of backward movement after brief exposure to blue light $(430-450 \mathrm{~nm})$. Young adult animals were observed by eye using a $10 \times$ objective and illuminated on the anterior half of the body during steady forward crawling on a clean agar surface. Typically $\sim 30$ trials were performed for each condition with no one worm tested more than $\sim 5$ times. Trials on individual animals were separated by $>2$ min to avoid habituation of the touch response.

\section{Results}

Disruption of RyR channels impedes both guidance and total regenerative outgrowth of laser axotomized neurons in vivo

Performing in vivo single-neuron laser axotomy of ALM mechanosensory neurons in live restrained adult $C$. elegans, we measured regenerative outgrowth (Fig. $1 A, B$ ) in wild-type animals and animals with mutations disrupting function of the $C$. elegans UNC-68/RyR channel (Maryon et al., 1996). As shown in Figure $1 C$, both unc-68(e540) and unc-68(r1162) mutants displayed an $\sim 60 \%$ decrease in average regenerative outgrowth of the ALM neuron $24 \mathrm{~h}$ after axotomy compared with wild-type. To assess whether UNC-68 is required within the damaged neuron to facilitate regeneration, we used strains expressing unc-68 in specific tissue of the unc-68(r1162) mutant (Liu et al., 2005). Consistent with a cell-intrinsic process, neuron-specific expression of UNC-68 fully rescued the regeneration defect, whereas expression in muscle tissue did not (no significant difference between muscle expression and unc-68(r1162); Figure 1C). We pharmacologically confirmed the unc- 68 mutant defect by treating animals with dantrolene, which blocks UNC-68/RyR channels and resulted in a decrease in regeneration (Fig. 1D). The smaller effect from dantrolene may reflect only partial efficacy of the drug compared with the genetic mutations. We performed regeneration experiments in two additional neuron types, the PLM and D-type motor neurons. Both resulted in significant regeneration defects in $u n c-68(e 540)$ mutants $24 \mathrm{~h}$ following laser axotomy (Fig. $1 E, F)$, suggesting that the result is valid across neuron type.

We further characterize the regeneration defects in $u n c-68$ mutants by performing continual time-lapse measurements in immobilized animals over the $5 \mathrm{~h}$ following laser axotomy. Wildtype neurons displayed a consistent increase in total outgrowth over this period, whereas outgrowth in unc-68(e540) animals ceased after the initial $\sim 1 \mathrm{~h}$ (Fig. $1 G$ ). As we have observed previously, the initial regenerative outgrowth consisted of small transient filopodial-like structures protruding from the damaged 
A

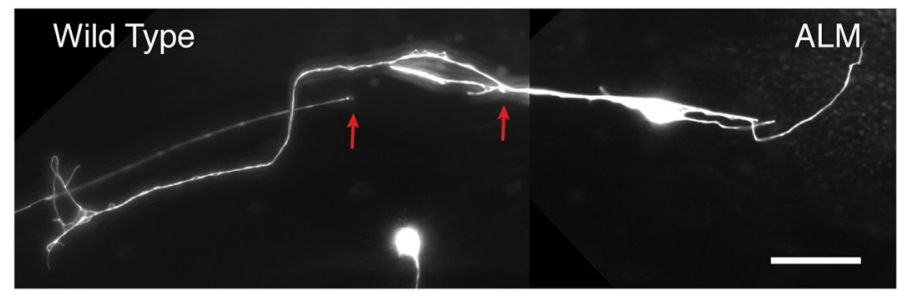

B

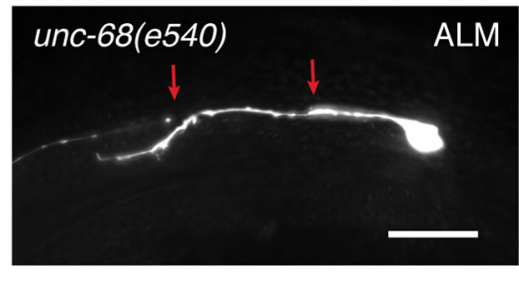

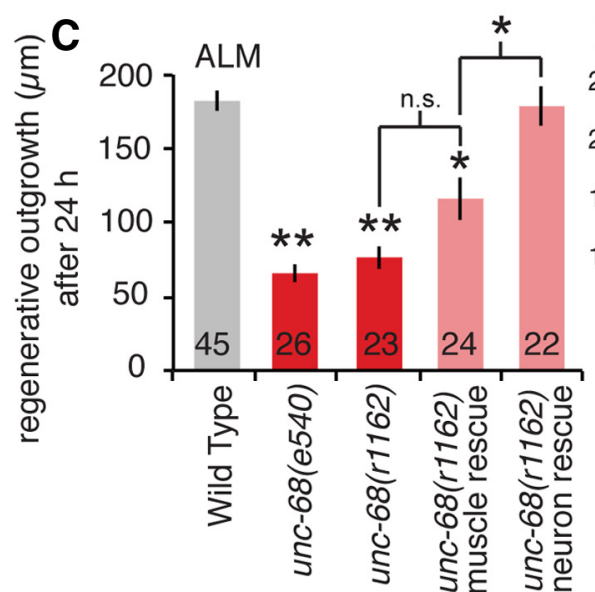

G

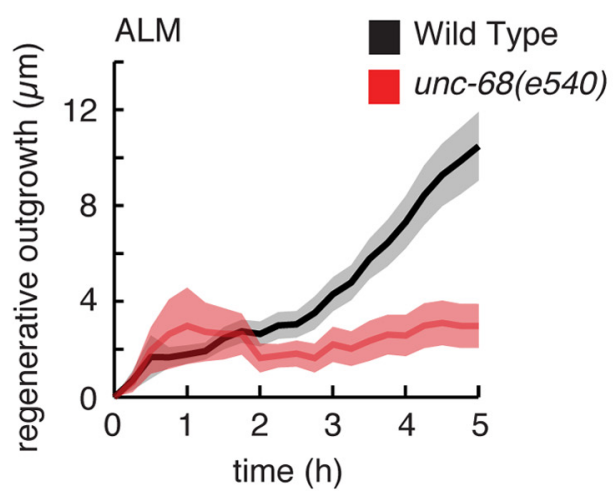

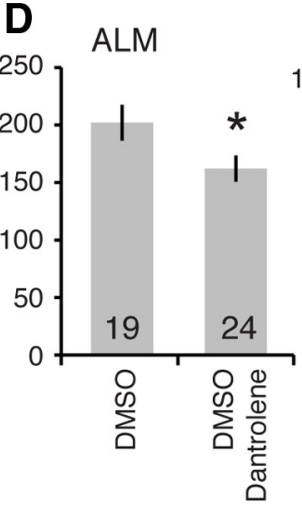

E PLM

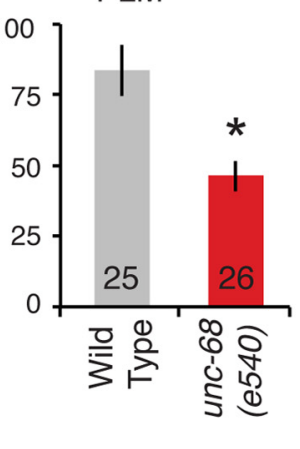

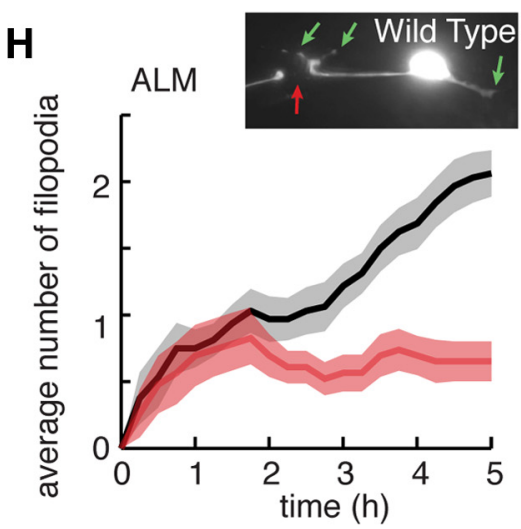

Figure 1. Disruption of RyR channels reduces in vivo neuronal regeneration. $\boldsymbol{A}, \boldsymbol{B}$, Example images of $C$. elegans ALM neuron regeneration at $24 \mathrm{~h}$ for wild-type and unc-68(e540) mutant worms. Red arrows indicate position of laser damage. Scale bars, $20 \mu \mathrm{m}$. C, Average regenerative outgrowth $24 \mathrm{~h}$ after laser axotomy of ALM neurons for wild-type, unc-68 mutants and rescue strains. ${ }^{*} p<$ 0.05 versus wild-type (except where indicated by the brackets). ${ }^{* *} p<0.001$ versus wild-type (except where indicated by the brackets). n.s., Not significant (multiple comparison in one-way AN0VA using a Tukey adjustment). $\boldsymbol{D}$, Average $24 \mathrm{~h}$ regenerative outgrowth of axotomized ALM neurons for control (with DMSO) and dantrolene (10 $\mu \mathrm{m}$ )-treated $C$. elegans. ${ }^{*} p<0.05$ ( $t$ test). $\boldsymbol{E}, \boldsymbol{F}$, Average $24 \mathrm{~h}$ regenerative outgrowth of axotomized PLM and D-type motor neurons for wild-type and unc-68(e540) mutants. ${ }^{*} p<0.05$ ( $t$ test). ${ }^{* *} p<0.001$ ( $t$ test). $\mathbf{G}$, Time-lapse measurement of average regenerative outgrowth for the $0-5 \mathrm{~h}$ following laser axotomy of ALM neurons. Measurements every $15 \mathrm{~min}$. Shaded region represents SEM. $\boldsymbol{H}$, The average number of filiopodial outgrowths in regenerating ALM neurons for wild-type and unc-68(e540) measured at every $15 \mathrm{~min}$ over the $0-5 \mathrm{~h}$ following laser axotomy. Inset, Example of wild-type regeneration at $5 \mathrm{~h}$ showing three filopodial outgrowths (green arrows).

neuron (Fig. $1 H$, inset). We quantified the number and timing of these structures (Fig. $1 H$ ) and found that, after generating an average of $\sim 1$ filopodial protrusion within the first hour, unc-68 neurons failed to increase the number of protrusions over the remaining $4 \mathrm{~h}$. This was in stark contrast to the steady increase in the number filopodia observed at longer time points in wild-type neurons.

RyR channels mediate attractive axon guidance signaling in cultured neurons by amplifying calcium gradients across an established growth cone (Hong et al., 2000). This illustrates the importance of continued RYR channel activity within a growing axon. We therefore tested whether the unc-68(e540) mutation affects axon guidance of regenerating $C$. elegans neurons in vivo. Following our previous study (Gabel et al., 2008), we severed the ventral axon projection of the C. elegans AVM mechanosensory neuron and measured the successful regeneration to the ventral nerve cord. We quantified guidance accuracy by scoring the percentage of neurons that successfully regenerated to the ventral nerve cord after $24 \mathrm{~h}$ and by measuring the "guidance angle" of each regenerating neuron. The guidance angle was defined as the angular difference between the regenerative outgrowth and the original axon path to the ventral nerve cord (Fig. $2 A, B$ ). As shown in Figure $2 C, D$, wild-type AVM neurons grew accurately with an average absolute guidance angle from the original path of $20.7 \pm 5.4^{\circ}$ and $90 \%$ of neurons successfully regrew to the ventral nerve cord after $24 \mathrm{~h}$. By contrast, unc- 68 mutant animals displayed a randomized AVM guidance angle with an absolute average value of $76.2 \pm 11.3^{\circ}$ ( $p<0.001$ by $t$ test) with only $28.6 \%$ successfully reaching the ventral nerve cord after $24 \mathrm{~h}$. Although, in some instances, regenerative outgrowth initiated 
A

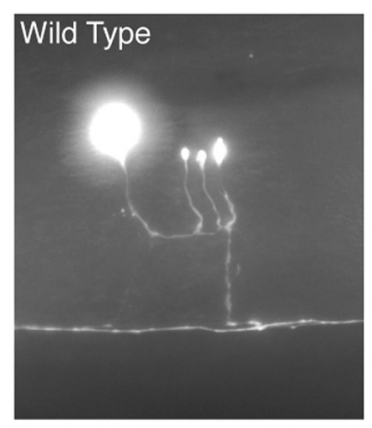

B
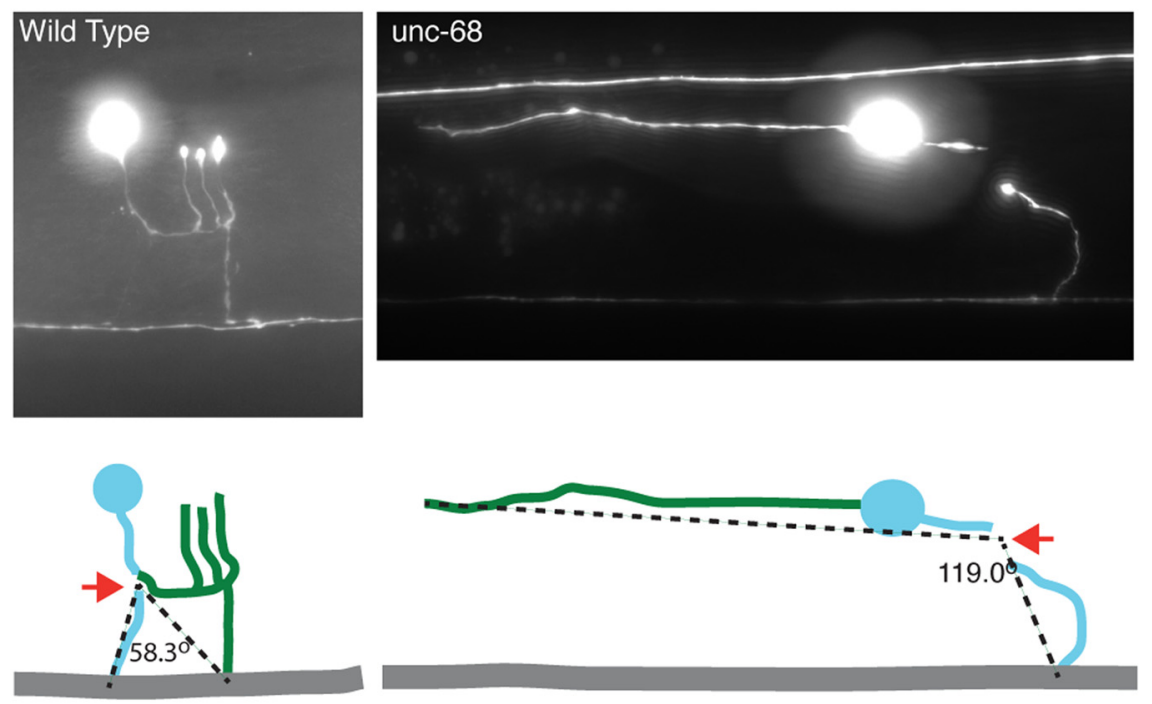

original neuron

ventral nerve cord

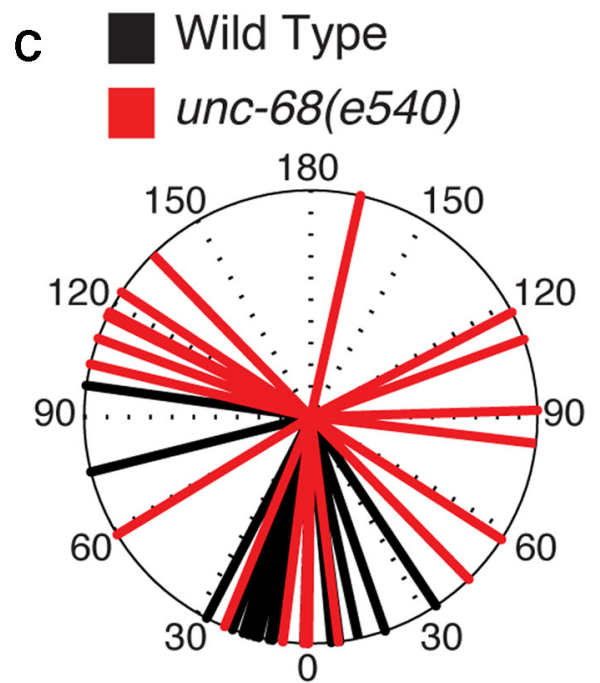

AVM guidence angle

\section{regeneration outgrowth}

laser damage point

Figure 2. Mutation of RyR channels disrupts axon guidance of regenerating neurons in vivo. $A, B$, Representative images of $24 \mathrm{~h}$ AVM regeneration for wild-type and unc-68(e540) mutant worms. Schematics show the original axon projection to the ventral nerve cord (blue), the laser damage point (red arrows), and the regenerative outgrowth (green). The axon guidance angle was measured as the angle between lines connecting the point of axotomy with the points where the original and new axons intersect the ventral nerve cord (or, if necessary, the end of the regenerative outgrowth). $\boldsymbol{C}$, Regeneration axon guidance angles of individual AVM neurons $24 \mathrm{~h}$ after axotomy for wild-type and unc-68(e540). D, Percentage of AVM neurons that successfully regrew to the ventral nerve cord $24 \mathrm{~h}$ after axotomy. ${ }^{* *} p<0.001$ by Fisher's exact test.

from the cell body (as in Fig. 2B), 90\% of wild-type and $75 \%$ of unc-68 mutant neurons regenerated from the severed end of the original axon (as in Fig. 2A, no significant difference by Fisher's exact test). Thus, the large defects we observe in unc$68(e 540)$ stem from poor guidance of the outgrowing neurite rather than differences in the point of initiation, suggesting that sustained RyR signaling actively guides regenerative growth over time.
RyR channels mediate sustained subcellular calcium signals in a damaged neuron that are critical in the initial stages of regeneration

To measure local subcellular calcium signals within a damaged neuron and how they might be altered in unc- 68 mutants, we laser axotomized neurons expressing genetically encoded calcium sensitive fluorophores. By laser severing ALM axons at a point $20 \mu \mathrm{m}$ from the cell soma and recording time-lapse images for 5 min, we first focused on the immediate intracellular calcium response to damage. Fluorescent measurements within the axon segment near the damage point (i.e., within $<5 \mu \mathrm{m}$ of the severed axon end) show a diminished calcium response immediately following axotomy (0-90 s) in unc-68 mutants compared with wild-type (Fig. 3A). However, the effects are most pronounced at later time points (after $\sim 200$ s) where wild-type neurons return to an elevated baseline level but this sustained calcium elevation is completely eliminated in the unc-68 mutants. Within the cell soma (Fig. 3B), we found similar but smaller defects in unc-68 mutants.

To measure intracellular calcium signals during the critical period when a damaged C. elegans neuron initiates new outgrowth and begins to regenerate, we extended our imaging assay over $5 \mathrm{~h}$ following laser surgery. Performing laser axotomies $60 \mu \mathrm{m}$ along the ALM axon, we measured calcium levels within $5 \mu \mathrm{m}$ of the severed axon end (or regenerating tip), the axon mid-point, and at the cell soma (Fig. 3C-E) at select time points. The longer cut distance results in no response within the cell soma (as observed previously; Kim et al., 2013) and a small immediate response at the axon midpoint, both of which appear unaltered in the unc-68 mutant. By contrast, close to the severed (or regenerating) axon end, unc68(e540) mutants show a significant decrease in the initial calcium response and complete elimination of sustained signals at longer times, $1-5 \mathrm{~h}$. This defect is reflected in the mean ratio of calcium signals at the axon end to those at the mid-point (Fig. 3D, inset). Representative ratiometric images illustrate the sustained calcium elevation near the axon end in wild-type but not unc- 68 mutants (Fig. 3F,G). These defects in calcium signaling correspond with the diminished outgrowth in unc-68 mutants over the same time period (Fig. 1G,H).

Periodic optogenetic photo-stimulation of a damage neuron enhances regenerative outgrowth

Given the above results, we asked whether it was possible to increase regeneration by dynamically increasing cellular calcium levels. For this, we used transgenically expressed ChR2, a light- 


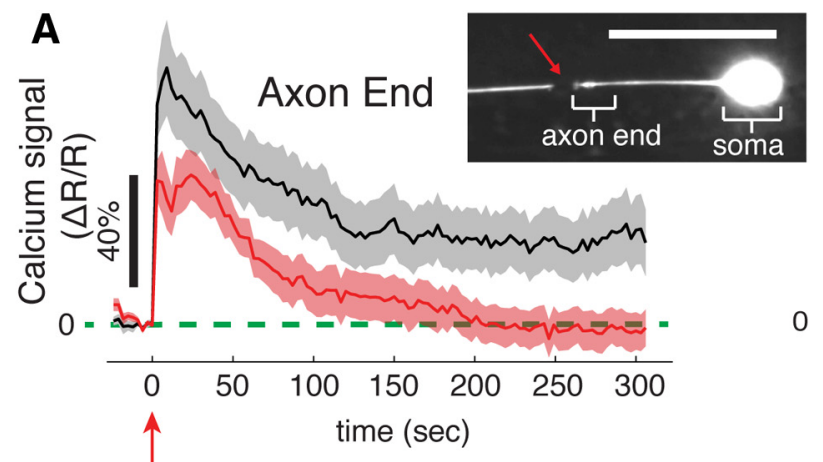

B
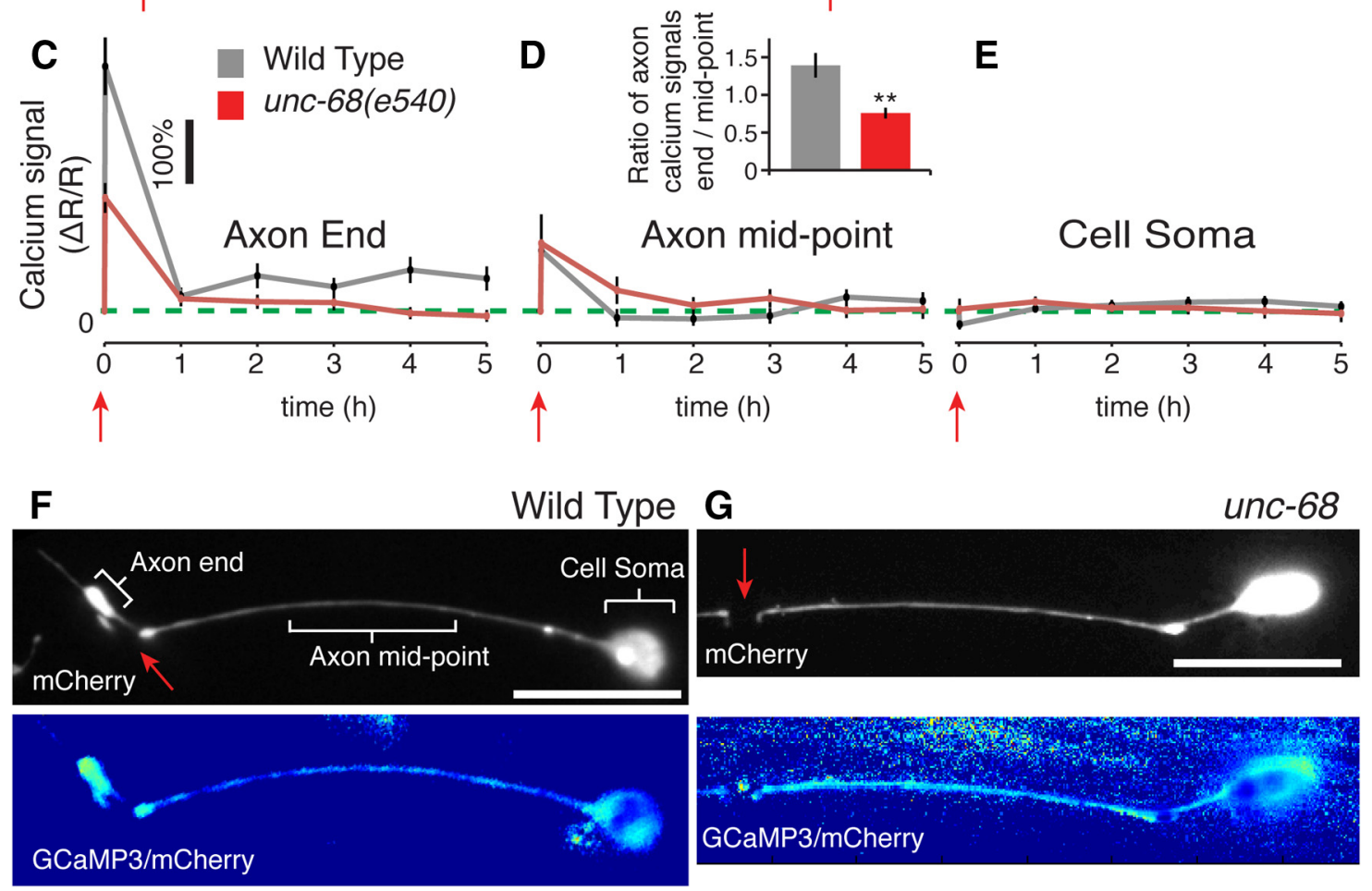

Figure 3. Subcellular calcium signals in a regenerating neuron. The localized average calcium signal within axotomized ALM neurons measured across the specified cellular regions of interests for $(\boldsymbol{A}, \boldsymbol{B})$ the immediate $0-300 \mathrm{~s}$ following laser axotomy $20 \mu \mathrm{m}$ from the cell soma or $(\boldsymbol{C}-\boldsymbol{E})$ the $0-5 \mathrm{~h}$ following laser axotomy $60 \mu \mathrm{m}$ from the cell soma. Shaded regions and error bars indicate SEM. $\boldsymbol{D}$, Inset, Ratio of calcium signals at the axon end to those at the mid-point averaged for data taken from 1 to $5 \mathrm{~h} .{ }^{* *} p<0.001$ ( $t$ test). $\boldsymbol{F}, \boldsymbol{G}$, Representative images showing subcellular calcium signaling in severed ALM axons for wild-type (at $4 \mathrm{~h}$ ) and unc-68(e540) (at $3 \mathrm{~h}$ ). Top, mCherry images alone. Bottom, Ratiometric images. Intensity indicates relative calcium levels (see Materials and Methods). Red arrows indicate time or position of laser damage. Scale bars, $20 \mu \mathrm{m}$.

activated cation channel that allows optical stimulation of neuron and muscle activity (Nagel et al., 2005). A $P_{\text {mec-4::ChR2::YFP; }}$ $P_{m e c-4}: \because m$ Cherry expressing strain allowed cell-specific imaging (560 nm mCherry excitation) and photo-activation $(470 \mathrm{~nm}$ ChR2 activation) of the mechanosensory neurons. At $1.5 \mathrm{~h}$ following laser axotomy, we exposed animals to continuous periodic (10 s on, $50 \mathrm{~s}$ off) photo-activation with uniform blue light. C. elegans do not endogenously produce ATR, the absorptive chromophore necessary for ChR2 response. It must be added to the growth media to enable ChR2 photo-activation, with animals lacking ATR functioning as controls for ChR2 expression and light exposure. As shown in Figure $4 A-D$, photo-stimulation of axotomized ALM neurons with $175 \mu \mathrm{W} / \mathrm{mm}^{2}$ blue light resulted in a $>30 \%$ increase in total regenerative outgrowth compared with controls at both 7 and $24 \mathrm{~h}$ after axotomy.

We found similar results performing experiments on the PLM neurons, which are a second pair of mechanosensory neurons in the tail of the animal. PLM neurons displayed greater ChR2 expression with brighter YFP labeling (YFP is fused to the $\mathrm{C}$ termi- nus of ChR2 creating ChR2::YFP to monitor expression levels) (Nagel et al., 2005). This often resulted in enlarge and malformed PLM cell somas and a marked decrease in $24 \mathrm{~h}$ regenerative outgrowth due to ChR2 expression (compare Wild Type to ChR2, ATR in Fig. 4E). Regardless, photo-stimulation of the PLM neurons with periodic (10 s on, $50 \mathrm{~s}$ off) blue light at $200 \mu \mathrm{W} / \mathrm{mm}^{2}$ increased regeneration by $\sim 50 \%$ over the nonstimulated ChR2, ATR controls (Fig. 4E).

Photo-stimulation of the touch neurons via ChR2 activation was verified by measuring a triggered reversal in response to $3 \mathrm{~s}, 1$ $\mathrm{mW} / \mathrm{mm}^{2}$ flashes of blue light illumination to the anterior half of freely crawling animals. Animals with ATR responded $\sim 80 \%$ of the time, whereas animals without ATR gave minimal response $(\sim 10 \%)$ (Fig. $4 F ; 0 \mathrm{~h}$ and No ATR data points). During the repetitive stimulus, we noted a reduction in response over time presumably due to bleaching of ATR. Exposing animals to the stimulating periodic light pattern for distinct time periods before performing the behavioral test resulted in a degradation of ChR2 response over the initial $6 \mathrm{~h}$ of periodic light exposure (Fig. $4 F$ ). 
A

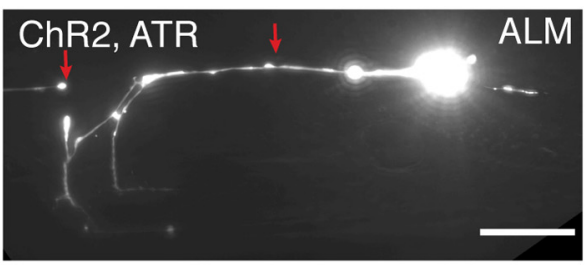

C

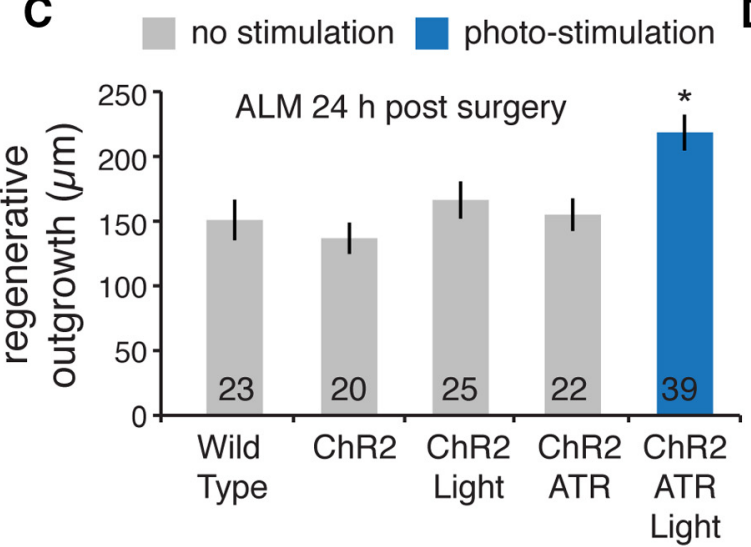

B

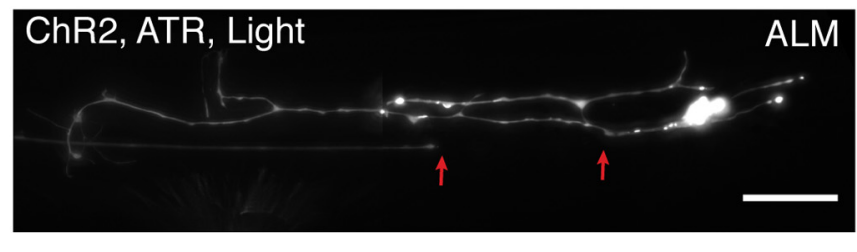

ALM $7 \mathrm{~h}$

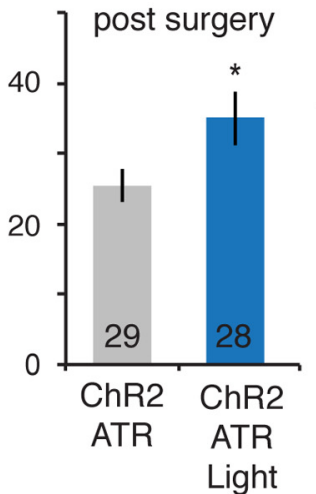

E

E PLM $24 \mathrm{~h}$ post surgery

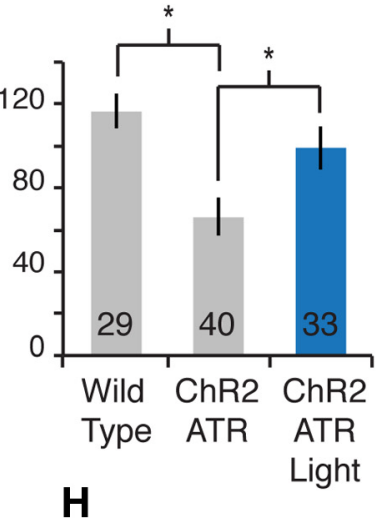

H

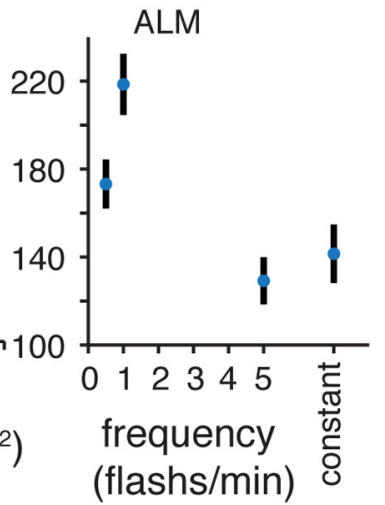

Figure 4. Periodic optogenetic stimulation enhances neuronal regeneration in vivo. $\boldsymbol{A}, \boldsymbol{B}$, Representative images of $24 \mathrm{~h}$ regeneration in nonstimulated (ChR2, ATR) and photo-stimulated neurons (ChR2, ATR, Light). Red arrows indicate position of laser damage. Scale bars, $20 \mu \mathrm{m}$. C, D, The average 24 and $7 \mathrm{~h}$ regenerative outgrowth in optogenetically stimulated ALM neurons exposed to repeating blue light ( 10 s on 50 s off, $175 \mu \mathrm{W} / \mathrm{mm}^{2}$ ) compared with nonstimulated controls. $\boldsymbol{E}$, Average $24 \mathrm{~h}$ regenerative outgrowth in control and optogenetically stimulated PLM neurons. $\boldsymbol{C}, \boldsymbol{E}$, ${ }^{*} p<0.05$ (multiple comparison in one-way ANOVA using a Tukey Adjustment). $\boldsymbol{D},{ }^{*} p<0.05$ ( $t$ test). $\boldsymbol{F}$, The behavioral response to a $3 \mathrm{~s}, 1 \mathrm{~mW} / \mathrm{mm}^{2}$ flash of blue light for animals exposed to the repeating photo-stimulation pattern used in the regeneration assays for the indicated time periods. Animals raised without ATR serve as controls. $\boldsymbol{G}, \boldsymbol{H}$, Average $24 \mathrm{~h}$ ALM regeneration with different light intensities $(\boldsymbol{G})$ and temporal patterns of photo-stimulation $(\boldsymbol{H})$.

Although it is not obvious that the threshold for the regenerative response is the same as that for behavior, we can confirm that damaged neurons were stimulated over this time period. Given that the enhance outgrowth was similar at 7 and $24 \mathrm{~h}$, it appears that much of the regenerative effect does indeed occur during this initial period.

The flexibility of the optically driven ChR2 stimulus allowed us to explore the dynamic response of the enhance outgrowth effect. Testing at various light intensities but with the same temporal exposure pattern (10 s on, $50 \mathrm{~s}$ off), we found that the enhanced outgrowth diminishes $<175 \mu \mathrm{W} / \mathrm{mm}^{2}$ for ALM neurons (Fig. 4G). Furthermore, altering the temporal pattern of excitation without changing the total light exposure, we found the maximal response for ALM neurons was at 1 exposure/min with the effect diminishing at faster or slower excitation rates (Fig. 4H). Thus, the specific dynamic characteristics of stimulation appear to be critical in eliciting a maximal regenerative response.
Interestingly, unc-68(e540) mutants or animals treated with dantrolene failed to show enhanced regeneration in response to ChR2 activation (Fig. $5 A$ ), suggesting a possible molecular mechanism for the effect. In C. elegans, muscle activation by ChR2 is dependent on both UNC-68/RyR channels and the $\alpha 1$-subunit of L-type voltage-gated calcium channels (EGL-19/VGCC) (Nagel et al., 2005). We found that nemadipine-A, which pharmacologically blocks the EGL-19/ VGCC channel (Kwok et al., 2006) and reduces regeneration in wild-type animals (Ghosh-Roy et al., 2010) and methods above, also eliminated the enhanced regenerative outgrowth resulting from ChR2 activation (Fig. $5 A$ ). It is important to note that expression of ChR2 appears to diminish the baseline regenerative defects in all conditions raising the possibility of confounding effects (see Materials and Methods). Nonetheless, in all cases, ChR2 activation (ChR2, ATR, Light) failed to enhance regeneration over the inactivated conditions, sug- 


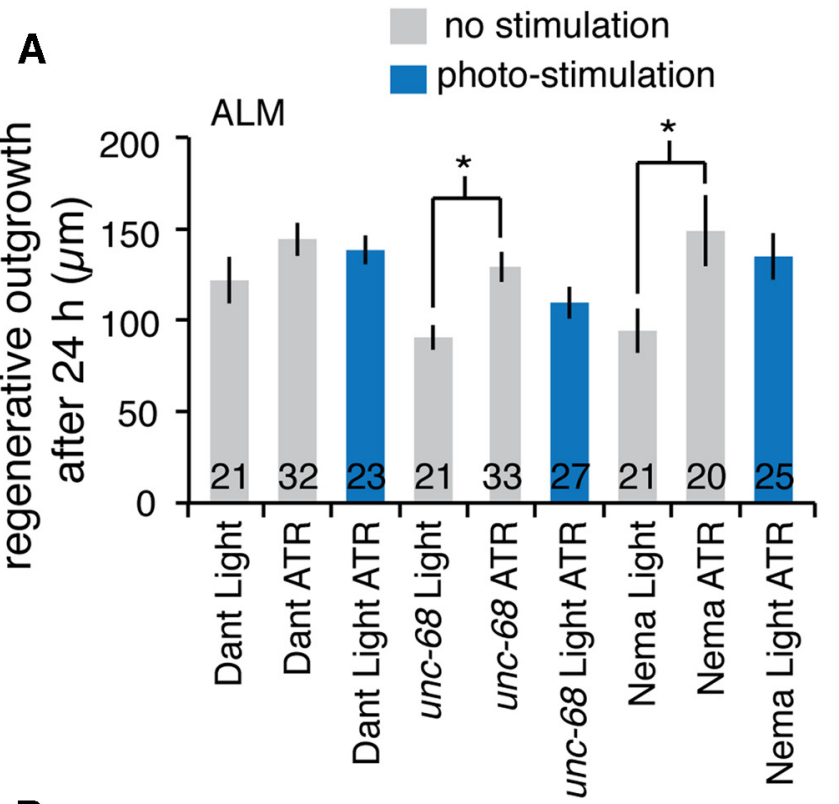

B

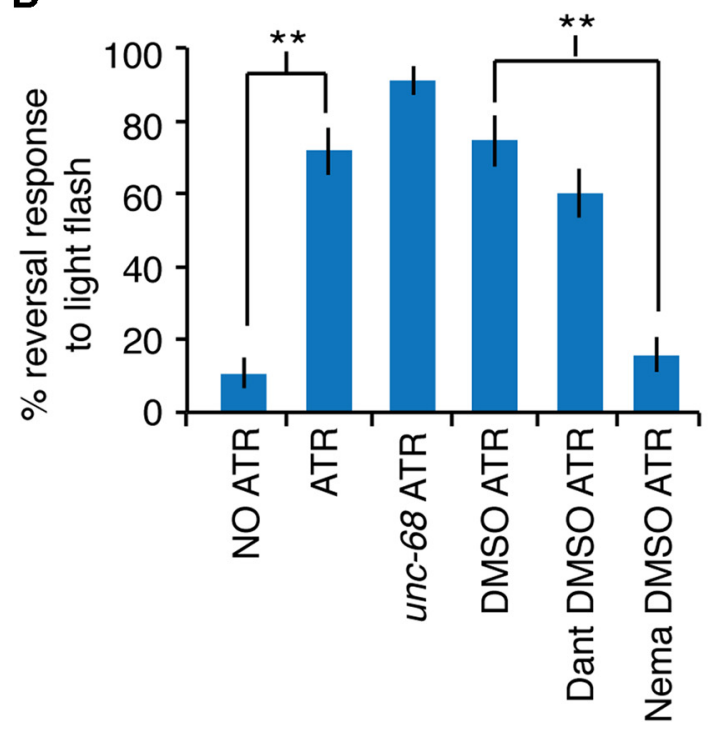

Figure 5. Disruption of UNC-68/RyR blocks enhanced regeneration of optogenetically stimulated neurons. $A$, Average $24 \mathrm{~h}$ ALM regeneration of photo-stimulated and control neurons in unc-68(e540), dantrolene-, and nemadipine-A-treated animals. ${ }^{*} p<0.05$ (multiple comparison in one-way ANOVA using a Tukey adjustment). $\boldsymbol{B}$, Percentage behavioral response to blue light stimulation for animals with the indicated conditions. Wild-type animals with NO ATR and animals treated with nemadipine-A responded significantly less than animals with ATR and DMSO ATR, respectively, compared through a logistic regression. ${ }^{* *} p<0.001$ (as indicated by brackets). More than 25 trials using $>5$ animals for each measurement.

gesting a ChR2, EGL-19/VGCC, UNC-68/RyR pathway may be at work to stimulate additional regenerative outgrowth.

Following previous studies demonstrating that electrical activation can enhance regeneration (Corredor and Goldberg, 2009), we investigated whether the enhanced regeneration from optogenetic stimulation was the direct result of the functional activity of the neuron. To do this, we measured the behavioral response to light exposure for unc-68(e540) mutants, as well as dantrolenetreated and nemadipine-A-treated animals (Fig. 5B). We found that $C$. elegans reliably respond with a triggered crawling reversal to ChR2-mediated ALM activation by a $1 \mathrm{~s}$ flash of $1 \mathrm{~mW} / \mathrm{mm}^{2}$ blue light but are unresponsive without the presence of ATR. Interestingly, whereas animals treated with nemadipine-A showed no behavioral response to light exposure, both unc-68(e540) mutants and dantrolene-treated animals displayed a wild-type response. This suggests that, although UNC-68/RYR mediates regenerative signals, it is not necessary for the function and presumably the underlying electrical activity of the ALM neuron in mediating behavior.

\section{Discussion}

Within an established growth cone, asymmetric calcium gradients control axon guidance, with attractive guidance cues stimulating vesicle exocytosis and outgrowth (Tojima et al., 2011). By amplifying this calcium gradient via CICR from the ER, RyR channels can mediate this attractive guidance response. Our results suggest that a similar mechanism may be at work following traumatic neuron damage, whereby localized calcium signals that are sustained and modulated via RyR channels mediate initial regeneration. We found that RyR signaling is important not only in accurate axon guidance (Fig. 2) but also modulates the amount of new regenerative outgrowth. This finding was reflected by the diminished outgrowth shown in Figure 1 where unc- 68 mutants show no growth or additional filopodia over the $1-4 \mathrm{~h}$ after axotomy of ALM neurons and a significant decrease in total regenerative outgrowth at $24 \mathrm{~h}$ in ALM, PLM, and D-type motor neurons. Expression of wild-type unc-68 specifically in the neurons of the unc-68(r1162) mutant successfully rescues the mutant phenotype, whereas expression in muscle tissue does not (Fig. $1 C)$. Thus, UNC-68/RyR appears to act cell autonomously to mediate axon regeneration across neuron type. In previous work on axon guidance in cell culture, RyR signaling determined the direction of axon outgrowth but did not affect total outgrowth rates (Hong et al., 2000). Thus, our findings add significant weight to the role of RyR signaling in regeneration showing that, within a damaged neuron, RyR calcium release is critical for efficient outgrowth.

By extending in vivo calcium imaging assays up to $5 \mathrm{~h}$ after axotomy, we measured sustained localized calcium signals within the damaged end of the severed axon as it begins to regenerate. These signals persist well beyond the large damage-induced calcium transients studied previously that last for only a few minutes following axotomy. The immediate signal is known to be important for efficient regeneration, is dependent on both external and internal calcium sources (Gitler and Spira, 1998; Chierzi et al., 2005; Ghosh-Roy et al., 2010), and modulates critical microtubule reorganization (Kamber et al., 2009). Here, we found that this immediate signal was partially dependent on UNC-68/RyR activity, as shown in Figure $3 A, C$. By contrast, the prolonged calcium signal over the $1-5 \mathrm{~h}$ following axotomy was completely eliminated in unc-68 mutants (Fig. 3 C). Although there remains the possibility of a complex calcium-triggered cascade whereby UNC-68/RyR is only active immediately following axotomy, evidence presented here along with previous knowledge of sustained RyR channel activity in axon guidance strongly suggests that RyR channels are the primary calcium source for the sustained, highly localized calcium elevation we observe at the regenerating end of severed axons. Indeed, the deficits in calcium signaling we measure correspond directly to the defects in regeneration. As seen in Figure $3 \mathrm{C}$ and Figure $1 \mathrm{G}, \mathrm{H}$, the unc-68(e540) mutation eliminated both regenerative outgrowth and calcium signals over the longer $1-5 \mathrm{~h}$ time period following axotomy, whereas the immediate calcium signal is only partially reduced and the immediate outgrowth $(0-1 \mathrm{~h})$ appears normal.

Within this context, we discovered that cell-specific ChR2 photo-stimulation of a damaged neuron can enhance regenerative outgrowth (Fig. 4). Genetic or pharmacological disruption of 
UNC-68/RyR activity appears to block this effect but does not alter the animal's behavioral, and presumably the underlying neuronal, response to ChR2 activation (Fig. 5). By contrast, we found that pharmacologically blocking L-type VGCCs eliminates both the behavioral and enhanced outgrowth response to ChR2 activation. In C. elegans muscle, ChR2 photo-activation is dependent on both EGL-19/VGCC and UNC-68/RyR activity, suggesting a mechanistic link between ChR2 activity and the physiological calcium response of the cell. Within a damaged neuron, ChR2 activation will depolarize the membrane, resulting in calcium influx via L-type VGCCs. The increased calcium could then trigger additional CICR from the ER via RyR channels, effectively amplifying the innate calcium signal to enhance regeneration. Although we have not directly measured the cytoplasmic calcium response to ChR2 activity, such a mechanism is consistent with ChR2 muscle activation in C. elegans, the known role of RyR in axon guidance, and our measurement of sustained calcium signals at the end of the regenerating axon. Importantly, within such a mechanism, it is the secondary cellular calcium signaling, rather than the direct electrical activity of the neuron, that triggers additional outgrowth. Interestingly, cultured rat retinal ganglion cells greatly increase their survival and outgrowth response to neurotrophic factors when exposed to a slow 1 min pattern of intermittent electrical activation (Goldberg et al., 2002), similar to the optimum pattern of photo-stimulation found here. Our results give new insights into these effects, emphasizing the importance of the underlying calcium signal.

The use of optogenetics in the context of neuronal damage and regeneration presents exciting new possibilities in basic regenerative biology. Numerous studies show that electrical stimulation can modify neuronal outgrowth in mammals (Corredor and Goldberg, 2009), but such results remain relatively limited given the physical constraints of the technique. By contrast, light activation of specific channels expressed in select cells allows a noninvasive, temporally and spatially dynamic stimulus administered to specific cells in vivo. Our experiments demonstrate the feasibility of this technique to beneficially alter neuronal regeneration and have begun to explore the optimal stimulation for enhancing regenerative outgrowth. In the future, the rapidly expanding array of optogenetic constructs, such as channelrhodopsin variants with improved light sensitivity and specific ion selectivity (Kleinlogel et al., 2011), will improve and refine the techniques presented here to allow highly specific control of single-cell physiology. Optogenetic strategies are actively being pursued for a range of possible therapies from restoration of vision (Doroudchi et al., 2011) to treatment of epilepsy (Paz et al., 2013). Our results illustrate its potential in the context of neuronal regeneration as well.

The specific source and spatiotemporal pattern of calcium dynamics within a damaged neuron can have widely differing effects on survival and regeneration depending on cellular context. For example, neuronal necrosis of $C$. elegans touch neurons in response to chronic calcium elevation is mediated in part by calcium release from the ER via UNC-68/RyR channels (Xu et al., 2001). Likewise, a recent study shows that axonal ER calcium release from RyR and $\mathrm{IP}_{3}$ channels is involved in degeneration of axons triggered by mechanical injury and toxic insults (Villegas et al., 2014). In mammalian cell culture, actively growing neurons are known to display an inverse relation between elongation rate and frequency of intracellular calcium transients (Gomez and Spitzer, 1999). Likewise, mammalian lesion-conditioned regeneration of DRG neurons appears to be triggered by a reduction of L-type calcium channel activity (Enes et al., 2010). On the other hand, activity-dependent calcium elevation promotes neuron survival during development (Mao et al., 1999) and calcium signaling resulting from brief patterns of electrical activity can enhance and modulate response to axon guidance cues (Ming et al., 2001) and increase outgrowth response to neurotrophic factors (Goldberg et al., 2002). Within the experiments presented here, we found defined thresholds for enhanced regeneration of stimulated neurons and a drastic effect on outgrowth based on changes in the temporal pattern of stimulation. Taken as a whole, these observations suggest that it will be critical to measure and locally manipulate cellular calcium in precise ways to accomplish therapeutic neuronal repair. Our work has identified a specific, localized calcium signal mediated by RyR channel calcium release that stimulates regenerative outgrowth. The state-of-the-art techniques we combined here open an exciting avenue of investigation to neurotherapeutic research, in which localized calcium manipulations could drive regeneration.

\section{References}

Chierzi S, Ratto GM, Verma P, Fawcett JW (2005) The ability of axons to regenerate their growth cones depends on axonal type and age, and is regulated by calcium, cAMP and ERK. Eur J Neurosci 21:2051-2062. CrossRef Medline

Chung SH, Sun L, Gabel CV (2013) In vivo neuronal calcium imaging in C. elegans. J Vis Exp 74. CrossRef Medline

Corredor RG, Goldberg JL (2009) Electrical activity enhances neuronal survival and regeneration. J Neural Eng 6:055001. CrossRef Medline

Doroudchi MM, Greenberg KP, Zorzos AN, Hauswirth WW, Fonstad CG, Horsager A, Boyden ES (2011) Towards optogenetic sensory replacement. Conference proceedings: Annual International Conference of the IEEE Engineering in Medicine and Biology Society IEEE Engineering in Medicine and Biology Society Conference 2011:3139-3141. CrossRef

Enes J, Langwieser N, Ruschel J, Carballosa-Gonzalez MM, Klug A, Traut MH, Ylera B, Tahirovic S, Hofmann F, Stein V, Moosmang S, Hentall ID, Bradke F (2010) Electrical activity suppresses axon growth through $\mathrm{Ca}(\mathrm{v}) 1.2$ channels in adult primary sensory neurons. Curr Biol 20:11541164. CrossRef Medline

Gabel CV, Antoine F, Chuang CF, Samuel AD, Chang C (2008) Distinct cellular and molecular mechanisms mediate initial axon development and adult-stage axon regeneration in C. elegans. Development 135:11291136. CrossRef Medline

Ghosh-Roy A, Wu Z, Goncharov A, Jin Y, Chisholm AD (2010) Calcium and cyclic AMP promote axonal regeneration in Caenorhabditis elegans and require DLK-1 kinase. J Neurosci 30:3175-3183. CrossRef Medline

Gitler D, Spira ME (1998) Real time imaging of calcium-induced localized proteolytic activity after axotomy and its relation to growth cone formation. Neuron 20:1123-1135. CrossRef Medline

Goldberg JL, Espinosa JS, Xu Y, Davidson N, Kovacs GT, Barres BA (2002) Retinal ganglion cells do not extend axons by default: promotion by neurotrophic signaling and electrical activity. Neuron 33:689-702. CrossRef Medline

Gomez TM, Spitzer NC (1999) In vivo regulation of axon extension and pathfinding by growth-cone calcium transients. Nature 397:350-355. CrossRef Medline

Hammarlund M, Nix P, Hauth L, Jorgensen EM, Bastiani M (2009) Axon regeneration requires a conserved MAP kinase pathway. Science 323:802806. CrossRef Medline

Hong K, Nishiyama M, Henley J, Tessier-Lavigne M, Poo M (2000) Calcium signalling in the guidance of nerve growth by netrin-1. Nature 403:93-98. CrossRef Medline

Kamber D, Erez H, Spira ME (2009) Local calcium-dependent mechanisms determine whether a cut axonal end assembles a retarded endbulb or competent growth cone. Exp Neurol 219:112-125. CrossRef Medline

Kim E, Sun L, Gabel CV, Fang-Yen C (2013) Long-term imaging of Caenorhabditis elegans using nanoparticle-mediated immobilization. PLoS One 8:e53419. CrossRef Medline

Kleinlogel S, Feldbauer K, Dempski RE, Fotis H, Wood PG, Bamann C, Bamberg E (2011) Ultra light-sensitive and fast neuronal activation with the $\mathrm{Ca}(2)+$-permeable channelrhodopsin CatCh. Nat Neurosci 14:513-518. CrossRef Medline 
Kwok TC, Ricker N, Fraser R, Chan AW, Burns A, Stanley EF, McCourt P, Cutler SR, Roy PJ (2006) A small-molecule screen in C. elegans yields a new calcium channel antagonist. Nature 441:91-95. CrossRef Medline

Liu K, Tedeschi A, Park KK, He Z (2011) Neuronal intrinsic mechanisms of axon regeneration. Annu Rev Neurosci 34:131-152. CrossRef Medline

Liu Q, Chen B, Yankova M, Morest DK, Maryon E, Hand AR, Nonet ML, Wang ZW (2005) Presynaptic ryanodine receptors are required for normal quantal size at the Caenorhabditis elegans neuromuscular junction. J Neurosci 25:6745-6754. CrossRef Medline

Mao Z, Bonni A, Xia F, Nadal-Vicens M, Greenberg ME (1999) Neuronal activity-dependent cell survival mediated by transcription factor MEF2. Science 286:785-790. CrossRef Medline

Maryon EB, Coronado R, Anderson P (1996) unc-68 encodes a ryanodine receptor involved in regulating C. elegans body-wall muscle contraction. J Cell Biol 134:885-893. CrossRef Medline

Ming G, Henley J, Tessier-Lavigne M, Song H, Poo M (2001) Electrical activity modulates growth cone guidance by diffusible factors. Neuron 29: 441-452. CrossRef Medline

Nagel G, Brauner M, Liewald JF, Adeishvili N, Bamberg E, Gottschalk A (2005) Light activation of channelrhodopsin-2 in excitable cells of Caenorhabditis elegans triggers rapid behavioral responses. Curr Biol 15: 2279-2284. CrossRef Medline

Paz JT, Davidson TJ, Frechette ES, Delord B, Parada I, Peng K, Deisseroth K, Huguenard JR (2013) Closed-loop optogenetic control of thalamus as a tool for interrupting seizures after cortical injury. Nat Neurosci 16:64-70. CrossRef Medline

Pinan-Lucarre B, Gabel CV, Reina CP, Hulme SE, Shevkoplyas SS, Slone RD,
Xue J, Qiao Y, Weisberg S, Roodhouse K, Sun L, Whitesides GM, Samuel A, Driscoll M (2012) The core apoptotic executioner proteins CED-3 and CED-4 promote initiation of neuronal regeneration in Caenorhabditis elegans. PLoS Biol 10:e1001331. CrossRef Medline

Schultheis C, Liewald JF, Bamberg E, Nagel G, Gottschalk A (2011) Optogenetic long-term manipulation of behavior and animal development. PLoS One 6:e18766. CrossRef Medline

Shin JE, Cho Y, Beirowski B, Milbrandt J, Cavalli V, DiAntonio A (2012) Dual leucine zipper kinase is required for retrograde injury signaling and axonal regeneration. Neuron 74:1015-1022. CrossRef Medline

Tojima T, Hines JH, Henley JR, Kamiguchi H (2011) Second messengers and membrane trafficking direct and organize growth cone steering. Nat Rev Neurosci 12:191-203. CrossRef Medline

Villegas R, Martinez NW, Lillo J, Pihan P, Hernandez D, Twiss JL, Court FA (2014) Calcium release from intra-axonal endoplasmic reticulum leads to axon degeneration through mitochondrial dysfunction. J Neurosci 34: 7179-7189. CrossRef Medline

Xu K, Tavernarakis N, Driscoll M (2001) Necrotic cell death in C. elegans requires the function of calreticulin and regulators of $\mathrm{Ca}(2+)$ release from the endoplasmic reticulum. Neuron 31:957-971. CrossRef Medline

Yan D, Jin Y (2012) Regulation of DLK-1 kinase activity by calciummediated dissociation from an inhibitory isoform. Neuron 76:534-548. CrossRef Medline

Yan D, Wu Z, Chisholm AD, Jin Y (2009) The DLK-1 kinase promotes mRNA stability and local translation in C. elegans synapses and axon regeneration. Cell 138:1005-1018. CrossRef Medline 